\title{
Time-Simultaneous Fan Charts: Applications to Stochastic Life Table Forecasting
}

\author{
W.S. Chan ${ }^{\mathrm{a}}$, J.S.H. Li ${ }^{\mathrm{b}}$ and A.C.Y. $\mathrm{Ng}^{\mathrm{a}}$ \\ ${ }^{a}$ The Chinese University of Hong Kong, Shatin, N.T., Hong Kong, P.R. China \\ ${ }^{\mathrm{b}}$ University of Waterloo, Waterloo, ON, Canada N2L $3 G 1$ \\ Email: chanws@cuhk.edu.hk
}

\begin{abstract}
When it comes to product pricing and reserving, actuaries often need life tables that include a forecast of future longevity improvement. However, the production of such tables is not straightforward, because the demographic future of any human population is a result of complex and only partially understood mechanisms, and is highly uncertain. In recent years, actuaries have been understandably concerned about error in the mortality assumptions they make. Part of their response is a new wave of work that is focused on the forecasting of uncertainty in longevity improvement, rather than producing a single mortality projection that will almost surely be wrong. This goal is accomplished by using stochastic mortality models, which have uncertainty embedded within them, as reflected in historical changes.

Given a fitted stochastic mortality model, we can express the uncertainty associated with future death rates in terms of confidence or prediction intervals. Recently, a group of researchers has proposed using fan charts to display prediction intervals for future mortality rates. These charts are highly parallel to the wellknown inflation fan charts, which have been produced periodically by the Bank of England since 1996. A fan chart depicts prediction intervals at different levels of confidence simultaneously. In particular, it shows the central $10 \%$ prediction interval with the heaviest shading, surrounded by the $20 \%, 30 \%, \ldots$, $90 \%$ prediction intervals with progressively lighter shading. We can therefore interpret the degree of shading as the likelihood of the outcome - the darker the shading, the more likely the outcome. Mortality fan charts are highly useful to actuaries, because they provide guidance on how to determine appropriate margins for adverse deviations.
\end{abstract}

Existing mortality fan charts are based on isolated pointwise prediction intervals. By pointwise we mean that the interval reflects uncertainty in a quantity at a single point of time, but it does not account for any dynamic property of the time-series. However, in actuarial practice, rather than a single death rate at a particular time point, what practitioners need is the entire trajectory of mortality rates for the birth cohort in question. Specifically, of their interest would be questions like "Within what bounds would the trajectory of cohort mortality rates likely to remain with a certain degree of confidence?" From a statistical viewpoint, a band of pointwise intervals might lead to invalid inference concerning the time trajectory. In particular, unless all trajectories develop very orderly, a band of pointwise confidence intervals would understate the actual uncertainty associated with a random mortality trajectory.

In this paper, we overcome this limitation by introducing the concept of time-simultaneous fan charts. In more detail, instead of pointwise intervals, a time-simultaneous fan chart is derived from a prediction band with a prescribed probability of covering the whole time trajectory. We present two numerical methods for producing time-simultaneous fan charts. These methods can be applied to common stochastic mortality models, including the generalized Cairns-Blake-Dowd model. We illustrate the method with mortality data from the populations of Australia and New Zealand.

Keywords: Cairns-Blake-Dowd model, Longevity risk, Prediction bands 


\section{INTRODUCTION}

When it comes to product pricing and reserving, actuaries often need life tables that include a forecast of future longevity improvement. However, the production of such tables is not straightforward. Tuljapurkar (2005) describes the challenge of forecasting mortality as "a bumpy road to Shangri-La," because the demographic future of any human population is a result of complex and only partially understood mechanisms, and is highly uncertain. Indeed, recent mortality data have unfolded significant deviations between the actual experience and the assumptions that actuaries made in the past (see, e.g., Continuous Mortality Investigation Bureau, 1999, 2002).

In recent years, actuaries have been understandably concerned about error in the mortality assumptions they make. Part of their response is a new wave of work that is focused on the forecasting of uncertainty in longevity improvement, rather than producing a single mortality projection that will almost surely be wrong. This goal is accomplished by using stochastic mortality models, for example, the Lee-Carter model (Lee and Carter, 1992) and its variants (e.g., Renshaw and Haberman, 2003, 2006; Delwarde et al., 2007; Li et al., 2009), which are fitted to historical data. The resulting models have uncertainty embedded within them, as reflected in historical changes. The use of such a stochastic approach is now highly regarded by leading actuarial organizations (see, e.g., Continuous Mortality Investigation Bureau, 2004).

Given a fitted stochastic mortality model, we can express the uncertainty surrounding a mortality projection in terms of confidence or prediction intervals. The interval estimates are crucially important to life insurers and annuity providers, since they provide guidance on how to determine appropriate margins for adverse deviations. Recently, Blake et al. (2008) and Dowd et al. (2010) propose expressing such intervals with mortality fan charts. A mortality fan chart depicts prediction intervals at different levels of confidence at the same time. In particular, it shows the central $10 \%$ prediction interval with the heaviest shading, surrounded by the $20 \%, 30 \%, \ldots, 90 \%$ prediction intervals with progressively lighter shading. We can therefore interpret the degree of shading as the likelihood of the outcome - the darker the shading, the more likely the outcome.

Existing mortality fan charts are based on isolated pointwise prediction intervals. By pointwise we mean that the interval reflects uncertainty in a quantity at a single point of time, but it does not account for any dynamic property of the time-series. However, in actuarial practice, rather than a single death rate at a particular time point, what practitioners need is the entire trajectory of mortality rates for the birth cohort in question. Specifically, of their interest would be questions like "Within what bounds would the trajectory of cohort mortality rates likely to remain with a certain degree of confidence?" From a statistical viewpoint, a band of pointwise intervals might lead to invalid inference concerning the time trajectory. In particular, unless all trajectories develop very orderly, a band of pointwise confidence intervals would understate the actual uncertainty associated with a random mortality trajectory.

In this paper, we overcome this limitation by introducing the concept of time-simultaneous fan charts. In more detail, instead of pointwise intervals, a time-simultaneous fan chart is derived from a prediction band with a prescribed probability of covering the whole time trajectory. We present two numerical methods for producing time-simultaneous fan charts. These methods can be applied to common stochastic mortality models, including the generalized Cairns-Blake-Dowd model. We illustrate the method with mortality data from the populations of Australia and New Zealand.

The rest of this paper is organized as follows. Section 2 describes the modeling of mortality dynamics. Section 3 presents two methods for constructing time-simultaneous mortality fan charts, and apply the methods to real mortality data. Finally, Section 4 concludes the paper with some recommendations to practitioners.

\section{Modeling Mortality Dynamics}

We use the generalized Cairns-Blake-Dowd model (Cairns et al., 2009) to model mortality dynamics. The model can be expressed as follows:

$$
\ln \left(\frac{q_{x, t}}{1-q_{x, t}}\right)=\kappa_{t}^{(1)}+\kappa_{t}^{(2)}(x-\bar{x})+\kappa_{t}^{(3)}\left((x-\bar{x})^{2}-\hat{\sigma}_{x}^{2}\right)+\gamma_{t-x}^{(4)},
$$


where $\kappa_{t}^{(1)}, \kappa_{t}^{(2)}$, and $\kappa_{t}^{(3)}$ are period risk factors, $\gamma_{t-x}^{(4)}$ is a cohort risk factor, and $\hat{\sigma}_{x}^{2}$ is the mean of $(x-\bar{x})^{2}$ over the age range we consider. The model can be estimated by the method of maximum likelihood.

Having fitted equation (1) to historical data, the period indexes $\kappa_{t}^{(1)}, \kappa_{t}^{(2)}$ and $\kappa_{t}^{(3)}$ are modeled by a trivariate random walk with drift, while the cohort index $\gamma_{t-x}^{(4)}$ is modeled by a first order autoregressive process. From these two processes, we can obtain a central projection and simulated sample paths of future death probabilities.

We consider the populations of Australian males and New Zealand males. The required data, death counts and exposures-to-risk on an annual basis, are obtained from the Human Mortality Database (2011). For Australia, we have data for years 1921 to 2007, and for New Zealand, we have data for years 1948 to 2008.

\section{Time-Simultaneous Mortality Fan Charts}

\subsection{Definitions}

Actuaries need cohort life tables for pricing and reserving purposes. Suppose that the forecast origin is year $T$. (We have $T=2007$ for Australia males and $T=2008$ for New Zealand males.) The cohort life table for an individual aged $x$ in year $T$ contains the following death probabilities:

$$
q_{x+1, T+1}, q_{x+2, T+2}, \ldots
$$

These death probabilities are not known as of year $T$ and have to be forecasted. We can communicate the uncertainty involved in the forecast with two types of prediction intervals.

Suppose that the forecast horizon, that is, the period for which the forecast is prepared, is $S$ years. We say $P I_{s}=\left[l_{s}, h_{s}\right]$ is a pointwise prediction interval for $q_{x+s, T+s}, s=1,2, \ldots, S$, with a coverage probability of $0<1-\alpha \leq 1$ if

$$
\operatorname{Pr}\left(l_{s} \leq q_{x+s, T+s} \leq h_{s}\right)=1-\alpha .
$$

A pointwise interval treats the underlying time-series random variable at different time points in isolation.

Denote by $\mathbf{q}=\left(q_{x+1, T+1}, q_{x+2, T+2}, \ldots, q_{x+S, T+S}\right)$ the entire trajectory of death probabilities over the forecast horizon. Unless all random trajectories develop very orderly, the probability that a trajectory lies completely inside all $S$ pointwise prediction intervals $P I_{s}, s=1, \ldots, S$, would be less than $1-\alpha$. Because actuaries care about the entire trajectory of death probabilities in most situations, mortality fan charts that are based on bands of pointwise prediction intervals are not sufficient in communicating the underlying uncertainty.

To improve the communication of uncertainty, we can consider a simultaneous prediction band. We say $\mathbf{P B}=[\mathbf{l}, \mathbf{h}]=\left(\left[l_{s}, h_{s}\right]\right)_{s=1}^{S}$ is a time-simultaneous prediction band for $\mathbf{q}$ with a coverage probability of $0<1-\alpha \leq 1$ if

$$
\operatorname{Pr}(\mathbf{q} \in \mathbf{P B})=\operatorname{Pr}\left(\bigcap_{s=1}^{S}\left(l_{s} \leq q_{T+s} \leq h_{s}\right)\right)=1-\alpha .
$$

In what follows, we describe two numerical methods for deriving time-simultaneous prediction bands from a stochastic mortality model. We then study how we may construct a mortality fan chart that is based on time-simultaneous prediction bands.

\subsection{Adjusted Intervals}

Using the stochastic mortality models described in Section 2, we can simulate realizations of the trajectory q. Let

$$
\mathbf{Q}=\left\{\mathbf{q}^{(n)}\right\}_{n=1}^{N}=\left\{\left(q_{x+1, T+1}^{(n)}, \ldots, q_{x+S, T+S}^{(n)}\right)\right\}_{n=1}^{N}
$$


be a sample of $N$ simulated trajectories. This sample is called the learning sample, which can be used to derive both pointwise and time-simultaneous prediction bands.

To derive a band of pointwise intervals, we first order the sample $\left\{q_{x+s, T+s}^{(n)}\right\}_{n=1}^{N}$, independently for each $s=1, \ldots, S$. Let $P I_{s}=\left[l_{s}, h_{s}\right]$ be a pointwise prediction interval for $q_{x+s, T+s}$ with a coverage probability of $1-\alpha$. Then, for each $s=1, \ldots, S$, the limits $l_{s}$ and $h_{s}$ are set to the $\lfloor N \alpha / 2\rfloor$ th and $\lceil N(1-\alpha / 2)\rceil$ th ordered values in the sample, respectively. Because the pointwise intervals are calculated by considering each death rate in isolation, the fraction of trajectories that are completely inside all $S$ pointwise prediction intervals $P I_{s}, s=1, \ldots, S$, is less than $1-\alpha$ in general.

From the learning sample, we can also construct a time-simultaneous prediction band which contains a randomly selected trajectory $\mathbf{q}^{(n)}$ in the learning sample $\mathbf{Q}$ with a probability of $1-\alpha$. We can accomplish this goal by two numerical methods proposed by Kolsrud (2007).

The first method is called 'adjusted intervals', which constructs a time-simultaneous prediction band by widening the pointwise intervals (with a pointwise coverage probability of $1-\alpha$ ) uniformly until the band of intervals has a simultaneous coverage of $1-\alpha$. In more detail, the method of adjusted intervals can be implemented with the following procedure.

1. For each $s=1, \ldots, S$, widen the interval uniformly to include the nearest sample point above and the nearest sample point below.

2. Check the simultaneous coverage of all intervals in the learning sample $\mathbf{Q}$.

3. If the simultaneous coverage is less than the prescribed level $1-\alpha$, go to Step (1). Otherwise, terminate the algorithm. The resulting band of intervals would contain no less than $1-\alpha$ of the trajectories in the learning sample. Note that the final coverage in the sample might be slightly larger than $1-\alpha$, because each uniform widening of all intervals includes at least two new trajectories.

\subsection{Chebyshev Bands}

The second method is called 'Chebyshev bands'. This method is based heavily on the concept of envelopes. The envelope of a (sub-)sample is the tightest band that contains all trajectories in the (sub)sample. For example, the envelope of the learning sample $\mathbf{Q}$ can be expressed as

$$
\left(\left[\min _{n} q_{x+s, T+s}^{(n)}, \max _{n} q_{x+s, T+s}^{(n)}\right]\right)_{s=1}^{S} .
$$

The method of Chebyshev bands constructs a time-simultaneous prediction band as the envelope of a subsample $\mathbf{Q}^{*}$ that contains $\lceil(1-\alpha) N\rceil$ trajectories with the shortest distance to the mean trajectory

$$
\overline{\mathbf{q}}=\left(\bar{q}_{x+1, T+1}, \ldots, \bar{q}_{x+S, T+S}\right),
$$

where $\bar{q}_{x+s, T+s}=\frac{1}{N} \sum_{n=1}^{N} q_{x+s, T+s}^{(n)}$ is the pointwise mean $s$ steps beyond the forecast origin.

The distance to the mean trajectory can be measured in different ways. For example, we can use the Chebyshev distance,

$$
\max _{s=1, \ldots, S}\left(\left|q_{x+s, T+s}-\bar{q}_{x+s, T+s}\right|\right),
$$

or the weighted Chebyshev distance,

$$
\max _{s=1, \ldots, S}\left(\frac{\left|q_{x+s, T+s}-\bar{q}_{x+s, T+s}\right|}{\sigma_{s}}\right),
$$

where

$$
\sigma_{s}=\sqrt{\frac{1}{N} \sum_{n=1}^{N}\left(q_{x+s, T+s}-\bar{q}_{x+s, T+s}\right)^{2}}
$$


is the pointwise standard deviation $s$ steps beyond the forecast origin. In this paper, the Chebyshev distance is used, because it takes into account the possible heteroskedasticity in the learning sample. This feature is important in our application since the volatility of the simulated death probabilities increases with both age and the distance from the forecast origin.

\subsection{Constructing the Fan Charts}

Given the methods for deriving time-simultaneous prediction bands, we can readily construct a timesimultaneous mortality fan chart by using the procedure below.

1. Plot the central projection of $\mathbf{q}$ with a solid line.

2. Construct a time-simultaneous prediction band for $\mathbf{q}$ with a coverage probability of $1-\alpha=0.1$. Shade the area between the upper and lower limits of the band.

3. For $i=2, \ldots, 9$, perform the following two steps:

(a) Construct a time-simultaneous prediction band for $\mathbf{q}$ with a coverage probability of $1-\alpha=$ $0.1 i$.

(b) Shade, using a lighter color, the area between the limits of the band with a coverage probability of $1-\alpha=0.1 i$ and the corresponding limits of the band with a coverage probability of $1-\alpha=0.1(i-1)$.

To illustrate, we consider the birth cohort who was aged 60 in year $T$, where $T=2007$ for Australian males and $T=2008$ for New Zealand males. The time-simultaneous mortality fan charts for the two populations are displayed in Figures 1 and 2. The fan charts shown are constructed with the method of Chebyshev bands. Those constructed with the method of adjusted intervals (not shown) are highly similar. Each fan chart shows the central 10\% time-simultaneous prediction interval with the heaviest shading, surrounded by the $20 \%, 30 \%, \ldots, 90 \%$ time-simultaneous prediction intervals with progressively lighter shading. The solid line in the middle of the 'fans' indicates the central projection.

Also shown in Figures 1 and 2 are the corresponding fan charts that are based on pointwise confidence intervals. These fan charts are significantly narrower than the corresponding time-simultaneous fan charts. In particular, at a prescribed coverage probability of $90 \%$, the pointwise mortality fan charts can only capture less than $50 \%$ of the trajectories in the corresponding learning samples. It is clear that pointwise mortality fan charts can seriously understate the uncertainty associated with q.

\section{Conclusions And Recommendations}

Because future mortality is difficult to predict, it is important to understand the uncertainty involved in making a mortality projection. Mortality fan charts can effectively present the degree of uncertainty surrounding the central mortality projection. As Blake et al. (2008) mention, they have a wide range of actuarial and financial applications, including pricing, hedging and setting capital requirements.

Nevertheless, mortality fan charts based on pointwise confidence intervals are inadequate, because they treat death probabilities at different time points in isolation. To overcome this limitation, we have introduced time-simultaneous mortality fan charts, which depict the uncertainty associated with the entire trajectory of future mortality rates. Such fan charts can be constructed easily with either one of the two numerical methods we presented.

Our illustrations indicate that the use of pointwise mortality fan charts can seriously understate the uncertainty surrounding a life table projection. Kolsrud (2007) conducted a deeper analysis of the coverage levels provided by pointwise prediction bands. It was found that at a prescribed coverage probability of $60 \%$, a band of pointwise confidence intervals can only capture $30.5 \%$ of the trajectories. The problem is even more serious at lower prescribed coverage probabilities. Therefore, in situations when the entire trajectory of the random quantity is important, a time-simultaneous fan chart should be used instead of a pointwise one.

The fan charts we produced incorporate the uncertainty arising from the stochastic nature of the assumed mortality model. However, they do not incorporate parameter uncertainty (i.e., the uncertainty involved 

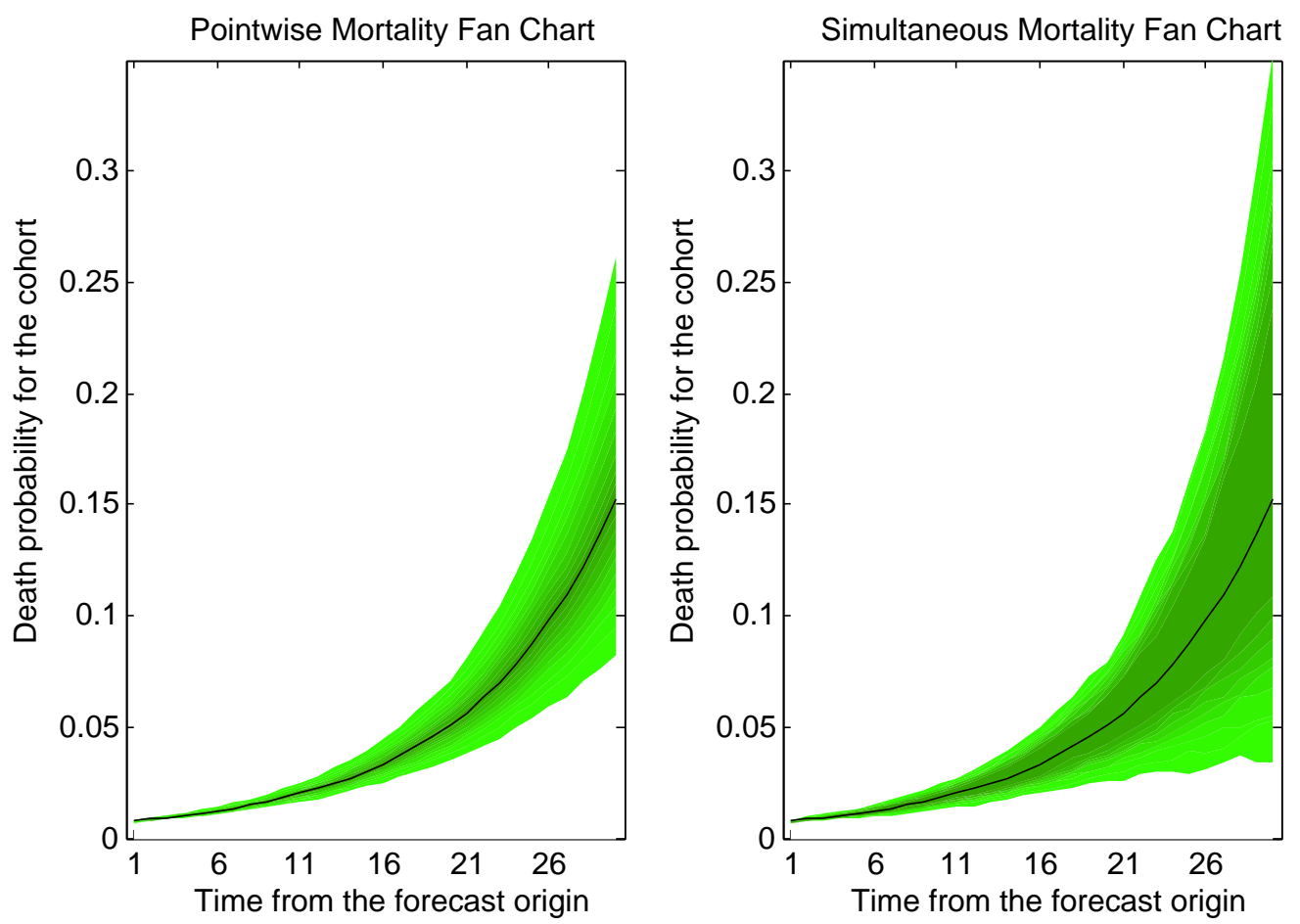

Figure 1. Pointwise and time-simultaneous mortality fan charts, Australian males
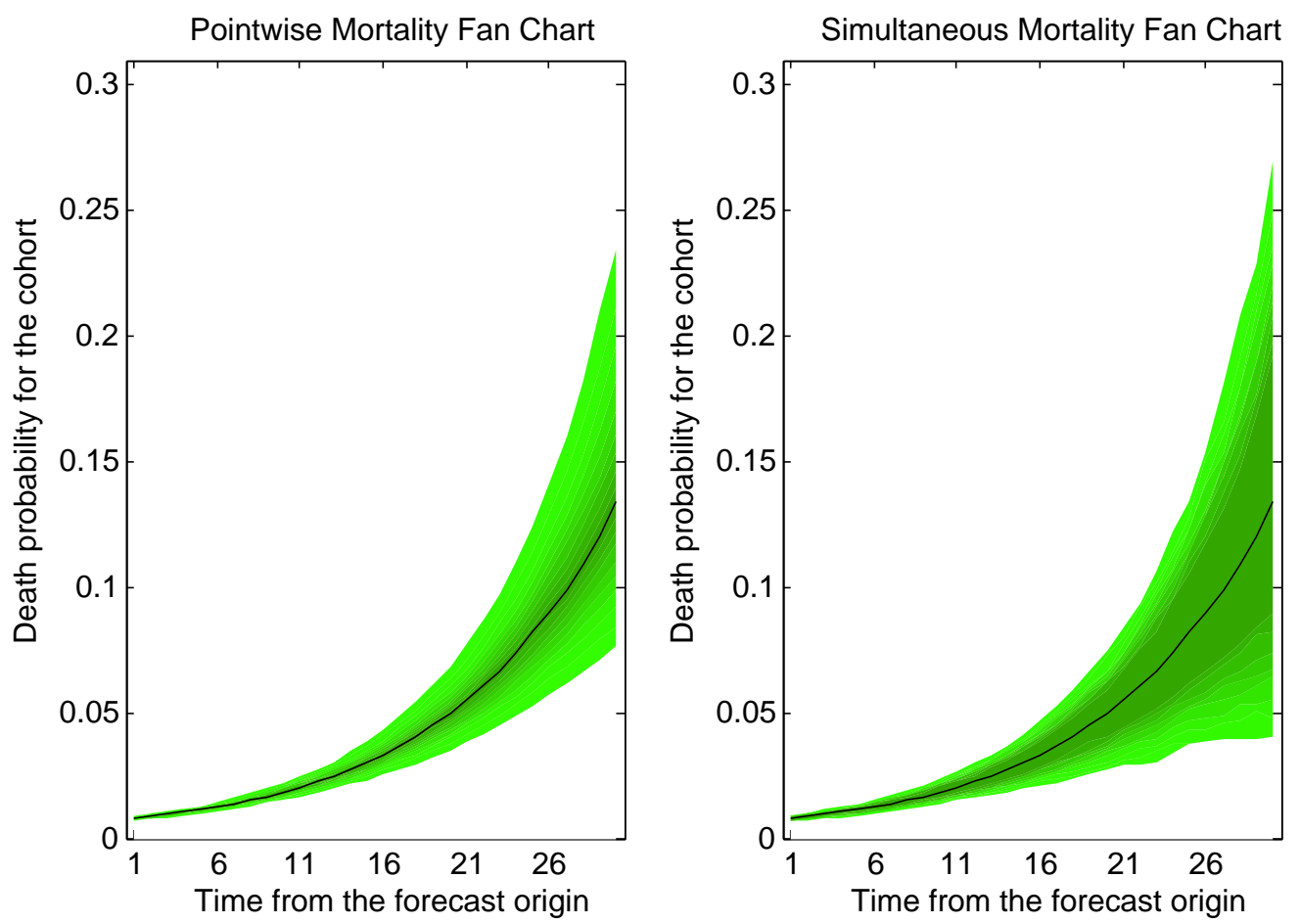

Figure 2. Pointwise and time-simultaneous mortality fan charts, New Zealand males 
in estimating the model parameters) and model uncertainty (i.e., the uncertainty in the model underlying what we are able to observe). Parameter uncertainty may be incorporated into the fan charts by Bayesian methods, but the quantification of model uncertainty is a lot more challenging and requires significant further research.

\section{ACKNOWLEDGMENTS}

This work was supported by a grant from the Natural Science and Engineering Research Council of Canada and by a direct grant from the Chinese University of Hong Kong (Project No. 2070401).

\section{REFERENCES}

Blake, D., A.J.G. Cairns, and K. Dowd (2008). Longevity risk and the Grim Reaper's toxic tail: the survivor fan charts. Insurance: Mathematics and Economics 42, 1062-1066.

Cairns, A.J.G., D. Blake, and K. Dowd (2006). A two-factor model for stochastic mortality with parameter uncertainty: theory and calibration. Journal of Risk and Insurance 73, 687-718.

Cairns, A.J.G., D. Blake, K. Dowd, G.D. Coughlan, D. Epstein, A. Ong, and I. Balevich (2009). A quantitative comparison of stochastic mortality models using data from England and Wales and the United States. North American Actuarial Journal 13, 1-35.

Continuous Mortality Investigation Bureau (1999). Standard tables of mortality based on the 1991-94 experiences. CMI Report no. 17. London: Institute of Actuaries and Faculty of Actuaries.

Continuous Mortality Investigation Bureau (2002). An interim basis for adjusting the "92" series mortality projections for cohort effects. CMI Working Paper 1. London: Institute of Actuaries and Faculty of Actuaries.

Continuous Mortality Investigation Bureau (2004). Projecting future mortality: a discussion paper. CMI Working Paper no. 3. London: Institute of Actuaries and Faculty of Actuaries.

Delwarde, A., M. Denuit, and C. Partrat (2007). Negative binomial version of the Lee-Carter model for mortality forecasting. Applied Stochastic Models in Business and Industry 23, 385-401.

Dowd, K., D. Blake, and A.J.G. Cairns (2010). Facing up to uncertain life expectancy: the longevity fan charts. Demography 47, 67-78.

Human Mortality Database. University of California, Berkeley (USA), and Max Planck Institute of Demographic Research (Germany). Available at www.mortality.org or www.humanmortality.de (data downloaded on 24 May 2011).

Kolsrud, D. (2007). Time-simultaneous prediction band for a time series. Journal of Forecasting 26, 171-188.

Lee, R. and L. Carter (1992). Modeling and forecasting U.S. mortality. Journal of the American Statistical Association 87, 659-671.

Li, J.S.H., M.R. Hardy, and K.S. Tan (2009). Uncertainty in mortality forecasting: an extension to the classical Lee-Carter approach. ASTIN Bulletin 39, 137-164.

Renshaw, A.E. and S. Haberman (2003). Lee-Carter mortality forecasting with age-specific enhancement. Insurance: Mathematics and Economics 33, 255-272.

Renshaw, A.E. and S. Haberman (2006). A cohort-based extension to the Lee-Carter model for mortality reduction factors. Insurance: Mathematics and Economics 38, 556-570.

Tuljapurkar, S. (2005). Future mortality: a bumpy road to Shangri-La? Science of Aging Knowledge Environment 14, pe9. 